\title{
Cohesive Properties and Asymptotics of the Dispersion Interaction in Graphite by the Random Phase Approximation
}

\author{
S. Lebègue, ${ }^{1}$ J. Harl, ${ }^{2}$ Tim Gould, ${ }^{3}$ J. G. Ángyán, ${ }^{1}$ G. Kresse, ${ }^{2}$ and J. F. Dobson ${ }^{3}$ \\ ${ }^{1}$ Laboratoire de Cristallographie, Résonance Magnétique et Modélisations (CRM2, UMR CNRS 7036) Institut Jean Barriol, \\ Nancy Université BP 239, Boulevard des Aiguillettes 54506 Vandoeuvre-lès-Nancy, France \\ ${ }^{2}$ Faculty of Physics and Center for Computational Materials Science, University of Vienna, Sensengasse 8/12, A-1090 Wien, Austria \\ ${ }^{3}$ Queensland Micro and Nano Technology Centre, Nathan campus, Griffith University, \\ 170 Kessels Road, Nathan, QLD 4111, Australia
}

(Received 21 June 2010)

\begin{abstract}
The structural properties of graphite, such as the interlayer equilibrium distance, the elastic constant, and the net layer binding energy, are obtained using the adiabatic-connection fluctuation-dissipation theorem in the random phase approximation. Excellent agreement is found with the available experimental data; however, our computed binding energy of $48 \mathrm{meV}$ per atom is somewhat smaller than the one obtained by quantum Monte Carlo methods. The asymptotic behavior of the interlayer dispersion interaction, previously derived from analytic approximations, is explicitly demonstrated to follow a $d^{-3}$ behavior at very large distances.
\end{abstract}

DOI:

PACS numbers: 71.15.Nc, 73.22.-f, 81.05.uf

Following the earlier interest in materials such as fullerenes and nanotubes, the interest in carbon-based materials has recently increased enormously since the routine production of graphene, a perfectly two-dimensional crystal made of carbon atoms, has become possible. Before that, graphite, the parent compound of this class of materials, has long been a focus of studies of both theoreticians and experimentalists. One intriguing issue is that, despite these efforts, the weak interplanar binding between graphene sheets (and more generally in all graphitic materials) is still poorly understood, mainly because it involves van der Waals interactions, which are notoriously difficult to describe within standard density functional theory. However, the precise description of this binding is of crucial importance, as it ultimately controls the structure and dynamics of all the systems related to graphite.

From the experimental point of view, the few available values were obtained indirectly, usually extracting the binding energy assuming simplified theoretical models. From a heat-of-wetting experiment, Girifalco and Lad [1] reported a value of $43 \mathrm{meV} /$ atom, while Benedict et al [2], performing measurements on collapsed nanotubes, obtained a value of $35 \pm 10 \mathrm{meV} /$ atom. More recently, Zacaria et al. [3] reported a larger value, $52 \pm 5 \mathrm{meV} /$ atom, by desorption of aromatic molecules from a graphite surface.

From the theoretical point of view, various methods were employed, ranging from semiempirical approaches $[4,5]$ to advanced first-principles calculations [6,7]. Since the standard approximations used in density functional theory (DFT) [8] such as the local density approximation (LDA) [9] or the generalized gradient approximation (GGA) [10] miss the long distance part of the van der Waals interaction, there were some attempts to add pairwise atom-atom corrections on top of the LDA/GGA calculations. Although these approaches are certainly able to cure part of the problem, their predictive power is limited. An alternative way that has been pursued recently is the van der Waals density functional (vdW-DF) [11-13], in which the correction taking into account the long-range part of the interaction is an explicit nonlocal functional of the density. Another approach [14], using second order perturbation theory combined with DFT, gives a binding energy value of 60-72 meV. But low order perturbation theory might overestimate correlation effects from low energy excitations, since MP2 diverges for metals. Very recently, Spanu, Sorella and Galli [7] have applied electronic quantum Monte Carlo (QMC) methods to determine the layer energetics of graphite and obtained a value of $56 \mathrm{meV} /$ atom.

Also, there is evidence from analytic theory [15-17] that the asymptotic form for the $\mathrm{vdW}$ interaction energy $U(d)$ between graphene layers is $U(d)=c_{3} d^{-3}$ at large interlayer separations $d$, as opposed to a simple $U(d)=c_{4} d^{-4}$ deriving from pairwise additive vdW interactions. This feature seems to be missed by all studies mentioned above, and raises the questions whether the magnitude of the $c_{3}$ coefficient is sufficiently small to neglect its contribution, or whether the previous calculations are simply not sufficiently converged with respect to the sampling of the Brillouin zone, resulting in the wrong asymptotic behavior.

Here, the adiabatic-connection fluctuation-dissipation theorem (ACFDT) [18-20] is used in the direct random phase approximation (RPA). There is substantial prior evidence [21-26] that this approach provides accurate results for isoelectronic energy differences-e.g. for the distance dependence of the energy of a combined system with fixed components separated by a variable distance $d$. In the RPA, the correlation energy is written as 


$$
E_{c}=\frac{1}{2 \pi} \int_{0}^{\infty} d \omega \operatorname{Tr}\left\{\ln \left[1-\chi^{\mathrm{KS}}(i \omega) v\right]+\chi^{\mathrm{KS}}(i \omega) v\right\}
$$

with $\chi^{\mathrm{KS}}$ being the independent particle (noninteracting) Kohn-Sham response function, and $v$ being the Coulomb kernel. The exchange energy is calculated exactly using the Hartree-Fock expression. All energies are evaluated using KS orbital of an initial calculation using the GGA-PBE functional [10]. The present computations were realized using the VASP code $[27,28]$ and the projector augmented wave method. Details and tests concerning the implementation of the ACFDT-RPA formalism within VASP can be found elsewhere $[25,26]$. To ensure the convergence of the binding energy, we used high plane wave cutoffs of $800 \mathrm{eV}$ for the Hartree-Fock part and $600 \mathrm{eV}$ for the correlation part. The response function was expanded in-plane waves up to an energy cutoff of $250 \mathrm{eV}$. For the correlation energy, the $k$-point sampling was performed using $14 \times$ $14 \times 6 k$ points for interlayer distances up to $4.5 \AA, 14 \times$ $14 \times 3 k$ points at intermediate interlayer distances, and $14 \times 14 \times 1 k$ points for the interlayer distances larger than $12 \AA$. The curves match smoothly onto each other. For the HF part, $26 \times 26 \times 8 k$ points were used. The $q$ variable in the independent particle response [25] $\chi_{\vec{G}, \vec{G}^{\prime}}^{\mathrm{KS}}(\vec{q}, i \omega)$ was sampled on the same fine grid. The inplane lattice parameter of graphite was not optimized but was set to the experimental value of $2.46 \AA$, while computations were realized for various values of the lattice parameter $c$ along the stacking direction ( $c$ corresponds to twice the layer distance with an $A B A B$ stacking sequence).

Our results for the equilibrium interplane distance $d_{0}$, the $\mathrm{C}_{33}$ elastic constant, and the binding energy are presented in the Table I and compared with results obtained using the local density approximation (LDA), the van der Waals density functionals [6,29], quantum Monte Carlo calculations [7], and with available experimental data. Let us first comment on the accuracy of the present results. The use of $14 \times 14 \times 3 k$ points for the entire energy-distance curve yields a binding energy of $50 \mathrm{meV}$, and decreasing the grid to $10 \times 10 \times 3 k$ points decreases the binding energy only slightly to $49 \mathrm{meV}$, suggesting that $10 \times 10 \mathrm{k}$ points are sufficient in the lateral direction. This is also confirmed by the observation that calculations using $10 \times 10 \times 5 k$ points yield a binding energy of $48 \mathrm{meV}$ identical to the reported value using
$14 \times 14 \times 6 k$ points. Hence, technically the present value is converged to about $1 \mathrm{meV}$.

Returning to Table I, we note that LDA, although purely local and therefore not able to describe dispersion interactions, gives a fairly good equilibrium lattice parameter, but underestimates the elastic constant by $30 \%$. The binding energy is also seriously underestimated in comparison with known experimental values. More interesting is the performance of vdW-DF for graphite [6,13] or two graphene sheets [29]. The best vdW-DFs gave binding energies reasonably close to our RPA values. However, even for the most refined variant of VdW-DF [6], the lattice parameter along the stacking direction is overestimated by about $10 \%$, and the $\mathrm{C}_{33}$ elastic constant is underestimated by at least $30 \%$. In contrast, the RPA provides equally good results for all essential structural and energetic parameters. Our calculated $d_{0}$ corresponds exactly to the experimental value, and the elastic coefficient $\mathrm{C}_{33}$ also matches the experimental values very well [34]. From these facts, it is clear that the description given by the RPA is better than the one of the commonly used vdW-DF, although certainly computationally significantly more expensive (for instance, for the equilibrium structure and with $14 \times 14 \times$ $6 k$ points, the RPA calculations typically required 5 hours on 64 cores). The situation is similar to that found by Marini et al. [24] for hexagonal boron nitride, where vdW-DF also overestimates $d_{0}$, while RPA gave a good agreement with experiment. Moreover, Spanu et al. [7] have applied electronic quantum Monte Carlo (QMC) methods to determine the layer energetics of graphite. They have obtained an equilibrium lattice parameter which is larger (3.426 $\AA$ ) than experiment and their overall layer binding energy is larger than ours $(56 \mathrm{meV} /$ atom versus our $48 \mathrm{meV} /$ atom). It is not yet clear whether the difference between their binding energy and ours is related to the small size of their simulation cell, corresponding to a rather coarse sampling of the Brillouin zone, or presents a genuine difference between RPA and DMC predictions. From our experience, however, it is likely that the DMC calculations may require additional effort in the size extrapolation, in order to obtain accurate values for the binding energy.

Another interesting quantity to be computed is the difference in energy between the two stacking sequences, $A B A B$ and $A A$. Using the ACFDT-RPA formalism, we

TABLE I. The equilibrium interplane distance $d$, the elastic coefficient $\mathrm{C}_{33}$, and the binding energy $E$ of graphite computed with the ACFDT-RPA formalism compared with results from LDA, VdW-DF, and QMC calculations, as well as with available experimental data.

\begin{tabular}{lccccc}
\hline \hline Method & LDA (Present) & VdW-DF $^{\mathrm{a}, \mathrm{b}}$ & QMC $^{\mathrm{l}}$ & ACFDT-RPA (Present) & Expts \\
\hline$d(\AA)$ & 3.33 & $3.76^{\mathrm{c}} 3.6^{\mathrm{a}} 3.59^{\mathrm{b}}$ & 3.426 & 3.34 & $3.34^{\mathrm{d}}$ \\
$\mathrm{C}_{33}(\mathrm{GPa})$ & 29.5 & $13^{\mathrm{c}} 27^{\mathrm{b}}$ & & 36 & $40.7^{\mathrm{e}} 36.5^{\mathrm{e}, \mathrm{f}} 38.7^{\mathrm{g}} 37^{\mathrm{h}}$ \\
$E(\mathrm{meV} / \mathrm{atom})$ & 24 & $24^{\mathrm{c}} 45.5^{\mathrm{a}} 50^{\mathrm{b}}$ & $56 \pm 5$ & 48 & $43^{\mathrm{i}}-35 \pm 10^{\mathrm{j}}-52 \pm 5^{\mathrm{k}}$ \\
\hline \hline
\end{tabular}

${ }^{\mathrm{a}}$ Ref. [29] ${ }^{\mathrm{b}}$ Ref. [6] ${ }^{\mathrm{c}}$ Ref. [13] ${ }^{\mathrm{d}}$ Ref. [30] ${ }^{\mathrm{e}}$ Ref. [31] ${ }^{\mathrm{f}}$ Ref. [32] ${ }^{\mathrm{g}}$ Ref. [33] ${ }^{\mathrm{h}}$ Ref. [34] ${ }^{\mathrm{i}}$ Ref. [1] ${ }^{\mathrm{j}}$ Ref. [2] ${ }^{\mathrm{k}}$ Ref. [3] ${ }^{\mathrm{l}}$ Ref. [7] 


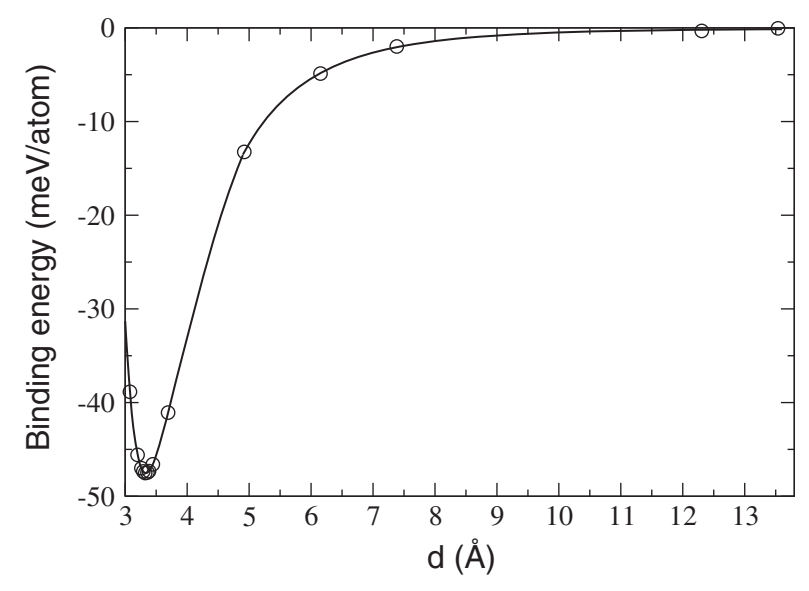

FIG. 1. Binding energy (in meV/atom) of graphite ( $A B$ stacking) as a function of the interplane distance (in $\AA$ ). The circles are the calculated points, the full line being only a guide to the eyes.

calculated it to be $10 \mathrm{meV} /$ atom, in favor of the $A B A B$ stacking. Our calculated equilibrium interplane distance for $A A$ graphite is $3.42 \AA$. In Ref. [35], the difference between the two stackings was explained by steric reasons: for the $A B$ stacking, the decrease in kinetic energy is larger than for the $A A$ stacking. Although this argument has been put forth in the framework of the local density approximation, it is still perfectly valid here.

Previous analytic work [15-17] predicts that the energy of highly stretched graphite (when the interplane distance approaches infinity) is of the form $E=$ const $+c_{3} d^{-3}$. This behavior is arising from $\pi_{z}-\pi_{z^{*}}$ transitions in the vicinity of the $k$ point. However, our present RPA calculations do not confirm this behavior upon first sight. A fit of the correlation energy from 3-9 $\AA$ yields an exponent $d^{-n}$ of $n=4.2$, very similar to the values reported before for DMC calculations [7]. A fit to distances between 9 and $24 \AA$ A however, suggests that the exponent might drop to values around 3 , but the results depend strongly on the applied $k$-point sampling and the considered distances. In order to elucidate this issue, we have performed additional calculations for a selected energy window around the Fermi level allowing only for transitions between $\pi_{z}$ and $\pi_{z *}$ states with a maximum energy transfer of $1.25 \mathrm{eV}$. Hence, all transitions involving other states than the $\pi_{z}$ bands crossing at the Dirac point $(\mathrm{K})$ are omitted. This reduces the computational effort massively, and we can apply a much denser $k$-point grid and consider significantly larger layer distances. Computing the correlation energy for a series of $k$-point grids, from $22 \times 22 \times 2$ to $32 \times 32 \times 2$ points, we find that all curves show a power law close to $d^{-3}$. For instance, for the $32 \times 32 \times 2$ set, the best fit is obtained for $f(d)=A+c / d^{2.84}$. The correlation energy for the grid of $32 \times 32 \times 2$ is shown in Fig. 2 visually yielding an excellent fit for $A+c_{3} / d^{3}$ and a poor description for $A+c_{4} / d^{4}$. This suggest that at large distances, in

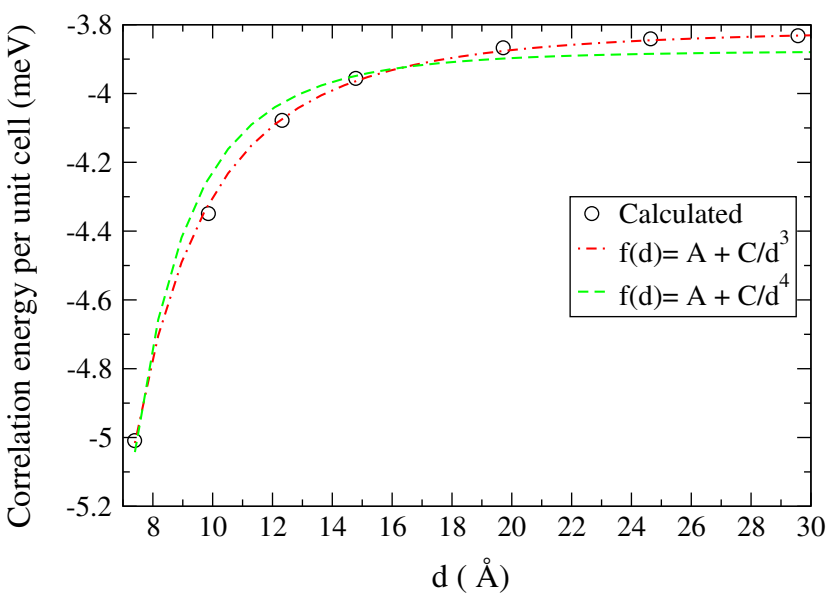

FIG. 2 (color online). Correlation energy (in meV per unit cell) at large interplane distance (in $\AA$ ) calculated from states close to the Fermi-level (see text). The calculated data (circles) are best fitted by a function with a $1 / d^{3}$ dependence (red dot-dashed) rather than a $1 / d^{4}$ dependence (green dashed).

fact, a $1 / d^{3}$ behavior is dominating; however the corresponding energy is small, of the order of a few meV only.

The predicted precoefficient $c_{3}$ is approximately $0.5 \mathrm{eV} \cdot \AA^{3}$ per unit cell, much smaller than from the analytical derivation [15-17], which suggested $c_{3}=$ $3.2 \mathrm{eV} \cdot \AA^{3}$ per unit cell. However, in the analytical studies, local field effects in the intraplane electronic screening were ignored, as was the finite bandwidth of the $\pi_{z}$ bands and the associated departure of the electronic Bloch wave dispersion from the linear form $E=v_{F}|\vec{k}-\vec{K}|$. Thus a perfect agreement of our calculations with the asymptotic theory cannot to be expected.

In view of the large number of $k$ points needed for convergence of RPA calculations on graphite due to its semimetallic behavior at the Fermi-level, it will be numerically challenging to extend our approach to more complex graphitic systems. More efficient algorithms are needed, perhaps with an explicit incorporation of the known analytic structure of graphitic response functions at small frequency and wave number [15]. In view of the limited $k$-point convergence of earlier calculations, as well as the spread of experimental data, we believe that our value of $48 \mathrm{meV} /$ atom for the graphite layer binding energy can be considered as the current benchmark against which new algorithms or functionals should be tested.

In summary, we have obtained the equilibrium lattice parameter, the elastic constant $\mathrm{C}_{33}$, and a benchmark layer binding energy for graphite, using the adiabatic-connection fluctuation-dissipation theorem in the random phase approximation. Excellent agreement was found in comparison with available experimental data for all three quantities. Our calculated binding energy differs by $15 \%$ from the one obtained from QMC, which is a reasonably small discrepancy, considering that the QMC calculations 
were performed using a rather small supercell. An important contribution of our work is the confirmation of the $c_{3} / d^{3}$ behavior of the correlation energy at large distances. Remarkably, the asymptotic behavior is not visible at short or medium distances, as it is completely overridden by the $1 / d^{4}$ behavior dominating at these distances. Further theoretical investigations will be required to understand why the $c_{3}$ coefficients are smaller than expected, and why the $1 / d^{3}$ behavior is not visible at all at small distances.

Supercomputer time was provided by GENCI (project $\mathrm{x} 2010085106)$ and the Vienna Scientific Cluster (VSC). This project is supported by the Australian Governments International Science Linkages program, and a FranceAustralia FAST grant. S. L. and J. G. A. acknowledge financial support from ANR Grant ANR-07-BLAN-0272 and ANR Grant ANR-06-NANO-053-02. J.H. and G. K. acknowledge financial support by the Austrian Fonds zur Forderung der wissenschaftlichen Forschung. J.D. acknowledges A. Rubio, P. Garcia-Gonzalez, G. Galli, I. Snook, and M. Per for useful discussions.

[1] L. A. Girifalco and R. A. Lad, J. Chem. Phys. 25, 693 (1956).

[2] L. X. Benedict, N. G. Chopra, M. L. Cohen, A. Zettl, S. G. Louie, and V.H. Crespi, Chem. Phys. Lett. 286, 490 (1998).

[3] R. Zacharia, H. Ulbricht, and T. Hertel, Phys. Rev. B 69 , 155406 (2004).

[4] L. A. Girifalco, M. Hodak, and R. S. Lee, Phys. Rev. B 62 , 13104 (2000).

[5] M. Hasegawa, K. Nishidate, and H. Iyetomi, Phys. Rev. B 76, 115424 (2007).

[6] E. Ziambaras, J. Kleis, E. Schröder, and P. Hyldgaard, Phys. Rev. B 76, 155425 (2007).

[7] L. Spanu, S. Sorella, and G. Galli, Phys. Rev. Lett. 103, 196401 (2009).

[8] P. Hohenberg and W. Kohn, Phys. Rev. 136, B864 (1964).

[9] W. Kohn and L. Sham, Phys. Rev. 140, A1133 (1965).

[10] J. P. Perdew, K. Burke, and M. Ernzerhof, Phys. Rev. Lett. 77, 3865 (1996).

[11] H. Rydberg, B. I. Lundqvist, D. C. Langreth, and M. Dion, Phys. Rev. B 62, 6997 (2000).
[12] H. Rydberg, M. Dion, N. Jacobson, E. Schröder, P. Hyldgaard, S. I. Simak, D.C. Langreth, and B. I. Lundqvist, Phys. Rev. Lett. 91, 126402 (2003).

[13] M. Dion, H. Rydberg, E. Schröder, D. C. Langreth, and B. I. Lundqvist, Phys. Rev. Lett. 92, 246401 (2004).

[14] Y. J. Dappe, M. A. Basanta, F. Flores, and J. Ortega, Phys. Rev. B 74, 205434 (2006).

[15] J. F. Dobson, A. White, and A. Rubio, Phys. Rev. Lett. 96, 073201 (2006).

[16] T. Gould, K. Simpkins, and J. F. Dobson, Phys. Rev. B 77, 165134 (2008).

[17] T. Gould, E. Gray, and J.F. Dobson, Phys. Rev. B 79, 113402 (2009).

[18] O. Gunnarsson and B. I. Lundqvist, Phys. Rev. B 13, 4274 (1976).

[19] D. C. Langreth and J. P. Perdew, Solid State Commun. 17, 1425 (1975).

[20] D. C. Langreth and J. P. Perdew, Phys. Rev. B 15, 2884 (1977).

[21] Z. Yan, J. P. Perdew, and S. Kurth, Phys. Rev. B 61, 16430 (2000).

[22] J.F. Dobson and J. Wang, Phys. Rev. B 62, 10038 (2000).

[23] J. Jung, P. Garcia-Gonzalez, J.F. Dobson, and R.W. Godby, Phys. Rev. B 70, 205107 (2004).

[24] A. Marini, P. Garcia-Gonzalez, and A. Rubio, Phys. Rev. Lett. 96, 136404 (2006).

[25] J. Harl and G. Kresse, Phys. Rev. B 77, 045136 (2008).

[26] J. Harl and G. Kresse, Phys. Rev. Lett. 103, 056401 (2009).

[27] G. Kresse and D. Joubert, Phys. Rev. B 59, 1758 (1999).

[28] G. Kresse and J. Furthmüller, Phys. Rev. B 54, 11169 (1996).

[29] S. D. Chakarova-Käck, E. Schröder, B. I. Lundqvist, and D. C. Langreth, Phys. Rev. Lett. 96, 146107 (2006).

[30] Y. Baskin and L. Meyer, Phys. Rev. 100, 544 (1955).

[31] W. B. Gauster and I. J. Fritz, J. Appl. Phys. 45, 3309 (1974).

[32] O. L. Blakslee, D. G. Proctor, E. J. Seldin, G. B. Spence, and T. Weng, J. Appl. Phys. 41, 3373 (1970).

[33] A. Bosak, M. Krisch, M. Mohr, J. Maultzsch, and C. Thomsen, Phys. Rev. B 75, 153408 (2007).

[34] N. Wada, R. Clarke, and S. A. Solin, Solid State Commun. 35, 675 (1980).

[35] J.-C. Charlier, X. Gonze, and J.-P. Michenaud, Europhys. Lett. 28, 403 (1994). 